${ }^{13} \mathrm{Van} \mathrm{Zyl}$, J. S., Annals. Eniv. Stellenbosch, 26 (1950).

is Poulanger, J., Ann. Geol. Madagascar, 26 (1959).

is Subramaniam, A. P., Bull. Geol. Soc. Amer., 67, 317 (1956).

${ }^{16}$ Leelanandam, C., Geol. Soc. India Bull., 4, 5 (1967).

${ }^{17}$ Lambert, R. St J., and Simons, J, G., Rappt. Gronlands Geol. Vuders., 19 $68(1969)$.

${ }^{13}$ Anderson, jun., A. T., Crewe, A. V., Goldsmith, J. R., Moore, P. B., Newton, J. C. Olsen, E. J., Smith, J. V., and Wyllie, 1.' J., Science, 167, 587 $(1970)$

${ }^{19}$ Wood, J. A., Marvin, U. B., Powell, B. N., and Dickey, jun., J. S., Smithson. Astrophys. Obs. Spec. Rep., 307, 37 (1970).

${ }^{20}$ Ghisler, M., and Windley, B. F., Rappt. Gronlands Geol. Unders.,12 (1967).

${ }^{21}$ Stueber, A. M., and Murthy, V. R., Geochim. Cosmochim. Acta, 30, 1243 (1966).

${ }^{22}$ Anhaeusser, C. R., Mason, R., Viljoen, M. J., and Viljoen, R. P., Eull. Geol. Stoe. Amer, 80, 2175 (1969).

${ }^{28}$ Soloolev, jun., N. V., Kuznetsova, I. K., and Zyuzin, N. I., J. Petrol., 9,

23 Hurley, P. M., and Rand, J. R. Science, 164, 1229 (1969).

\section{Anomalous Propagation of Elastic Energy within the Moon}

THe records of the seismographs left on the lunar surface by the Apollo 11 and Apollo 12 missions have been discussed by Latham et al. ${ }^{1}$. From the behaviour immediately following impact of the Apollo 12 ascent stage and similar events present on the rccords before and after, they have inferred that energy propagation as elastic waves over large distances can be represented as a diffusive process in a low loss elastic continuum. They calculate a diffusion coefficient of $\sim 1 \mathrm{~km}^{2} \mathrm{~s}^{-1}$ and a specific dissipation function $Q \sim 3,600$ at a frequency of $1 \mathrm{~Hz}$. If we use the time delay between the Apollo 12 impact and the commencement of the signal as the propagation time of an unscattered wave, we deduce a wave velocity of $\sim 3 \mathrm{~km} \mathrm{~s}^{-1}$ and a scattering length $\sim 0.5 \mathrm{~km}$. They emphasize that such a medium is quite unknown on Earth.

We wish to point out a few observations that encourage one to pursue this model for the Moon:

(i) The experiments of one of us (B. I. P.) on the measurement of $Q$ in porous terrestrial rocks consistently show that $Q$ increases when the ambient air pressure is reduced At $\sim 10^{-2}$ torr, the limit of evacuation in these expori ments, $Q$ is typically five timcs greater than at atmospheric pressure. The effect is reversible and subsidiary experiments suggest that it is connected with an exchange of water $<0.05$ per cent by weight. Although indirect arguments ${ }^{2}$ have been givon that the lunar rocks did not acquire this amount of water under laboratory conditions, we suspect that the laboratory $Q$ values of lunar rocks obtained by Kanamori et $a l^{3}(\sim 10)$ at one atmosphere may not bo representative. Obviously it will be necessary to make $Q$ measurements on lunar rocks that have been kept under high vacuum.

(ii) The experiments of Grossman et al. ${ }^{4}$ domonstrate that the surfaces of Iunar material cleft under ultra high vacuum adhere when placed in contact. If the in situ scattering length just referred to is taken as the typical lincar dimensions of separate blocks, the normal stresses present at the contact points would be more than adequate to maintain adhesion at the stress levels of the seismic wave.

(iii) Orowan ${ }^{5}$ has suggested that stress waves through a medium may have rapidly increasing $Q$ as the stress amplitude tends to zcro. He suspects that if relativo displacements of opposing crack faces approach interatomic distanees the effective friction decreases to zero. This idea is perhaps not distinct from the concept of adhosion mentioned under (ii) (the maximum amplitude of the Apollo 12 signal was $\sim 30 \AA$ ).

Perhaps the most important factor to cmphasize is that although $Q$ appears as a property of the medium in a continuum theory, from a structural point of view, if the material is an aggrogate, the overall $Q$ is sensitive to the state of the surfaces that make contact. Merely to postulate that the near surface region of the Moon is such an effective scattering medium probably ensures that no gas pressure can build up within it to destroy the adhesiveness of the bearing surfaces. The concept of the lossless "welded" contact mentioned by Latham et al. ${ }^{1}$ seems to have some empirical support in this particular environment.

We thank J. C. Savage who supervised the experiments mentioned in section (i).

B. I. Pandit

D. C. TOZER

Department of Geophysies,

University of Toronto,

Toronto,

Canada.

Received February 27, 1970.

${ }^{1}$ Latham, G. V., Ewing, M., Press, F., Sutton, G., Dorman, J., Nakamura, Y. 'Toksöz, N., Wiggins, R., Derr, J., and Duennebier, E., Science, 16\%, 455 $(1970)$.

${ }^{2}$ Friedman, I., O'Neil, J. R., Adami, I. H., Gleason, J. D., and Hardcastle, K., Science, $167,538(1970)$.

${ }^{3}$ Kanamori, H., Nur, A., Chung, D., Wones, D., and Simmons, G., Seience, $167,726(1970)$

Grossman, J. J., Ryan, J. A., Mukherjee, N. R., and Wegner, M. W. Science, 16\%, 743 (1970).

"Orowan. E., Non-Elastic Processes in the Mantle (edit. by Tozer, D. C.), 196 (Blackwell, 1967).

\section{Quasars behind Clusters of Galaxies}

A systematic search for quasi-stellar objects associated with clusters of galaxies was started by Sandage and Miller ${ }^{1}$, but was discontinued when no cluster was found near 3C 48. The search has been resumed recently by Bahcall, Schmidt and Gunn ${ }^{2}$. Bahcall ${ }^{3}$ found twenty-one QSOs with redshifts larger than $z=0.2$ in the direction of Zwicky clusters. In all these cases the redshifts of the QSOs are considerably larger than the redshifts of the associated clusters. Assuming that the redshifts are cosmological, all the twenty-one QSOs would be at considerable distances behind the clusters. This suggests a simple explanation of the association of clusters with QSOs; namely, that QSOs are gravitational lens images*.

The bending of light in the gravitational field of astronomical objects is a well established phenomenon. The recognition of the possible existence of gravitational lenses can be traced as far back as 1919 , when Lodgo ${ }^{5}$ noted that a gravitational lens does not have a real focal length and produces two images. All the papers published before 1965 discuss the effect in Euclidean space, with the deflecting mass confined within a radius which is small compared with the distance by which the light rays bypass the deflector. The solution in non-Euclidean space for the situation when the light rays pass through the mass distribution of the deflector and for relativistic cosmological models was worked out by J. M. B. ${ }^{4}$ and J. M. B. and M. F. B. ${ }^{6}$. Recently, Saslaw, Faulkner and Strittmatter ${ }^{7}$ as well as ourselves ${ }^{8}$ applied the gravitational lens effect to explain pulsating radio sources.

Every galaxy is a potential gravitational lens for all objects proparly lined up behind it. The foreground galaxy acting as the lens will produce a greatly distorted image, but in amplified brightness. The image has the shape of two thin erescents, which may coalesce to a thin ring. A small galdxy of $10^{10} M_{\odot}$ amplifies by more than five magnitudes the brightness of any object which happens to lie within $2 \times 10^{-8} \mathrm{rad}$ of the line of sight through its gravitational contre. From Wagoner's ${ }^{9}$ calculations we know that to a redshift of $z=2$ we see 3 to 15 per cent of all galaxies through a foreground galaxy. While not all of these intervening galaxies will be properly aligned with a suitable object to produce a large brightness amplification, the presence of a large number of gravi- 